\title{
(Musik)instrument (im) Computer
}

\author{
Miriam Akkermann, Dresden
}

DOI: $10.25366 / 2020.102$

Zitation: Miriam Akkermann, "(Musik)instrument (im) Computer", in: Brückenschläge zwischen Musikwissenschaft und Informatik. Theoretische und praktische Aspekte der Kooperation, in Verbindung mit der Fachgruppe Digitale Musikwissenschaft hrsg. von Stefanie Acquavella-Rauch, Andreas Münzmay und Joachim Veit (= Musikwissenschaft: Aktuelle Perspektiven. Bericht über die Jahrestagung der Gesellschaft für Musikforschung 2019 in Paderborn und Detmold, Bd. 3), Detmold, Musikwissenschaftliches Seminar der Universität Paderborn und der Hochschule für Musik Detmold, 2020, S. 125-140, DOI: 10.25366/2020.102

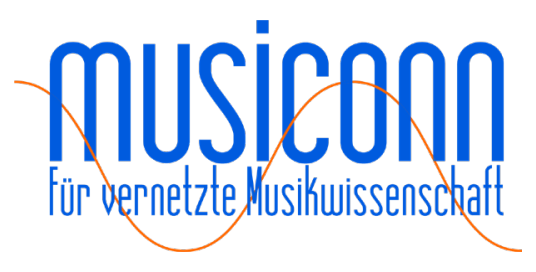




\title{
(Musik)Instrument (im) Computer
}

\author{
MIRIAM AKKERMANN, DRESDEN
}

Der Einsatz von Computern ist in aktuellen Musikproduktionen allgegenwärtig und kaum mehr wegzudenken. Dies gilt für die Popmusik ebenso wie für weite Teile der klassischen Musik. Computer werden im Entstehungs- wie Aufführungsprozess an unterschiedlichen Stellen mit verschiedenen Zielen eingesetzt. Die Möglichkeiten reichen von Notensatzprogrammen über das MIDI-gesteuerte Abspielen von Samples bis hin zu individuell programmierten KlangsyntheseProgrammen, Musikprogrammiersprachen zur Steuerung oder Klangerzeugung, oder der digitalen Nachbearbeitung von Aufnahmen. Diese heute scheinbar so selbstverständlichen Einsatzbereiche entstammen einer noch relativ jungen Entwicklungsgeschichte und gehen weit über die beiden Hauptaufgaben hinaus, für die Computer in ihren Anfängen in der Musik eingesetzt wurden, nämlich Partitursynthese, das Berechnen und Erstellen von Notation nach Regeln der Komponist*in und Klangsynthese (Klangberechnung) bzw. Klangveränderung. Das Zusammenspiel von Hard- und Software sowie ihre gegenseitige Einflussnahme ziehen sich hierbei nicht nur durch die Entwicklungsgeschichte der Technologien und damit auch der Klangsynthese, sondern werfen auch grundlegende Fragen in der Betrachtung von Musikstücken wie -instrumenten auf, bei deren Entwicklung oder Umsetzung entsprechende Technologien genutzt wurden.

\section{Technische Vorgeschichte zur Klangsynthese in Schlaglichtern}

Wie eng die Entwicklung von Technologien mit der Exploration neuer Möglichkeiten digitaler Klangerzeugung verwoben ist, zeigt ein Blick auf die Geschichte der Computermusik. Einer der Pioniere in der Computermusik war Max Mathews (1926-2011). Er entwickelte 1957 MUSIC I, eines der ersten Programme, die es ermöglichten, Klang basierend auf beliebigen Wellenformen mittels Computer zu generieren. ${ }^{1}$ Hierfür nutzte er einen IBM 704, dem nach Mathews Aussage einzigen vor Ort vorhandenen Computer, mit dem es zu diesem Zeitpunkt möglich war, sound processing durchzuführen. ${ }^{2}$ Diese Berechnungen erfolgten dabei nicht in Echtzeit, da die benötigte Rechenzeit oft mehrere Stunden betrug. Mathews arbeitete an den Bell Laboratories, einer privaten Forschungseinrichtung in Murray Hill, New Jersey. Die nach Alexander Graham Bell benannten Bell Laboratories entstanden als Forschungsgruppe des amerikanischen TelefonMonopolisten American Telephone and Telegraph Company (AT\&T) in einem Joint Venture mit

1 Vgl. Tae Hong Park, „An Interview with Max Mathews”, in: Computer Music Journal 33/3 (2009), S. 9-22, hier S. 11, <https://doi.org/10.1162/comj.2009.33.3.9>.

2 Vgl. Curtis Roads und Max Mathews, „Interview with Max Mathews”, in: Computer Music Journal 4/4 (Winter 1980), S. 15, <https://doi.org/10.2307/3679463>. 
Western Electric und waren trotz ihres Status als private Forschungseinrichtung durch staatliche Maßnahmen vor Wettbewerb geschützt. Dann kam - für fast eine Dekade - zunächst der offiziell erstmals 1959 ausgelieferte IBM 7090, sowie der IBM 7094 (Abb. 1) an den Bell Laboratories zum Einsatz, die Mathews nach eigener Aussage für MUSIC II (1958), MUSIC III (1960) und MUSIC IV

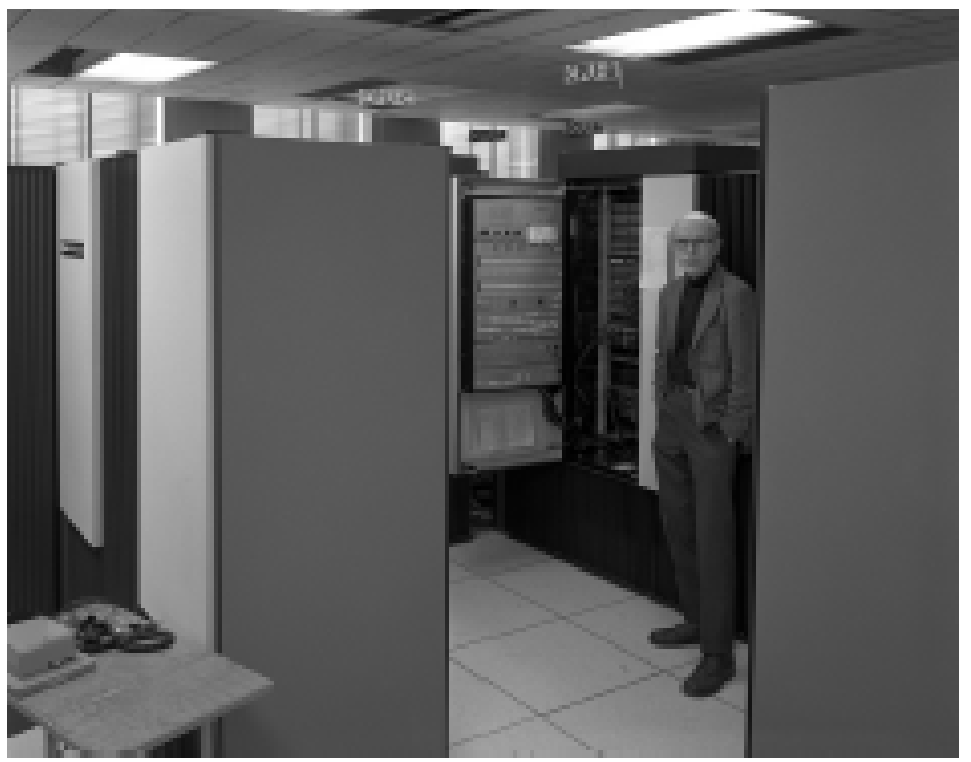

Abbildung 1: Max Mathews vor IBM 7094 Mainframe Computer, 1965, Bell Laboratories (The Oxford Handbook of Computer Music, hrsg. von Roger T. Dean, Oxford u. a. 2009, S. 19)

(1963) nutzte. Das Design erlaubte es, mit dem Austausch weniger Bauteile einen IBM 7090 auf einen IBM 7094 aufzurüsten, sowie alle bereits vorhandenen Programme nun mit höherer Geschwindigkeit auszuführen. ${ }^{3}$ Die neuen Versionen von Matthews Programms umfassten nicht zwangsläufig mehr Kapazität zur Klangerzeugung, sondern entstanden auch, um einfachere oder bessere Steuerungsmöglichkeiten zu bieten oder die grundlegende Programmierung zu verbessern. Insbesondere die Implementierung von Makro-Assembler in das bisher rein in Assembler Code geschriebene Programm war ein technischer Zugewinn, wie Mathews schildert: ${ }^{4}$

"Macro assemblers were just invented at that time. Indeed, Music IV debugged a lot of the macro assembler that was used at Bell Labs. It made very heavy and rather sophisticated use of macro facilities, and I discovered a lot of bugs in them that the designers hadn't anticipated, and that they were glad to fix."

3 Vgl. ebd., S. 16, sowie IBM Archives, "7090 Data Processing System”, <https://www.ibm.com/ibm/history/exhibits/mainframe/mainframe_PP7090.html> und "7094 Data Processing System", $<$ https://www.ibm.com/ibm/history/exhibits/mainframe/mainframe_PP7094.html> (beide 20.08.2020). Für das Upgrade von IBM 7090 auf IBM 7094 wurden der zentrale Prozessor (IBM 7100 Modell 2), die Steuerungskonsole (IBM 7151 Modell 2) und ein Teil des IBM 7606 Multiplexer (7094 Feature \#7146) getauscht. Dies führte zu einer teilweise erheblichen Leistungssteigerung, so konnte der neue Prozessor beispielsweise zwei Befehle pro Kernspeicherzyklus bearbeiten. Neue Funktionen des IBM 7094 waren: Gleitkommaoperationen mit doppelter Genauigkeit, sieben Indexregister und neue Befehle zur Indexkomplementierung (vgl. ebd.).

4 Vgl. Roads und Mathews, "Interview”, S. 17. 
Je besser die Programme wurden, umso mehr fanden sie Verbreitung. Einer der ersten Orte hierbei war das Center for Computer Research in Music and Acoustics (CCRMA) an der Stanford University, zu dem Mathews Kontakte hielt. Dort nutzten die Komponisten John Chowning und David Poole im Jahr 1964 MUSIC IV, um Klänge auf einem IBM 7090 zu generieren, einem Rechner, der in Stanford ab 1962 vorhanden war und der dasselbe grundlegende Betriebssystem wie der IBM 7094 nutzte, was eine Übertragung von MUSIC IV auch auf das Vorgängermodell ermöglichte. ${ }^{5}$

Eine Recheneinheit, die für die Erzeugung von Klängen in Echtzeit eine wichtige Rolle spielte, war der sogenannte VAX 11/780 der Firma DEC. Dieser kam 1977 auf den Markt und setzte als erster im Handel erhältlicher 32-bit-Computer eine neue Hardware-Benchmark für die CPU-Leistung, die als VAX MIPS bezeichnet wurde, da das Modell als eine sogenannte MIPS (One Million Instructions Per Second) Machine galt. ${ }^{6}$ Damit bot dieses (wie schon die Rechner der PDP-Familie) zur Klasse der Minicomputer gehörende Modell eine verlässliche technologische Grundlage für die Entwicklung rechenintensiver Klangsyntheseverfahren. Im Jahr 1980 wurde beispielsweise für diesen Rechner Cmusic entwickelt, eine Programmiersprache für Klangsynthese, die direkt auf der Recheneinheit auch ausgeführt werden konnte. ${ }^{7}$ Am MIT wurde für die VAX 11 die Version MUSIC 11 entwickelt, eine nun in C programmierte Version der MUSIC Reihe, die für alle C-basierten Mikroprozessoren verwendbar war. Denn es gab zwar mit MUSIC 5 und MUSIC 360 bereits C-basierte Versionen, diese waren jedoch auf Forschungszentren mit zentralisierten Computing-Systemen ausgelegt. ${ }^{8}$ Zudem war die VAX 11 in den 1980er Jahren an den auf diesem Gebiet führenden Institutionen vorhanden, z. B. an der University of California San Diego (spätestens ab 1982) sowie am Institut de Recherche et Coordination Acoustique/Musique (IRCAM) in Paris (spätestens ab 1986). ${ }^{9}$

5 Vgl. Paul Doornbusch, „Early Hardware and Early Ideas in Computer Music: Their Development and Their Current Forms", in: The Oxford Handbook of Computer Music, hrsg. von Roger T. Dean, Oxford u. a. 2009, S. 44-84, hier S. 47; Robert Braden, "Burroughs Algol at Stanford University, 1960-1963”, in: IEEE Anals of the History of Computing, 2013, S. 69-73, hier S. 71; Jack Harper, „IBM 7090/94 Architecture”, unter <http://www.frobenius.com/7090. htm> (15.01.2020), sowie Miriam Akkermann, Zwischen Improvisation und Algorithmus, David Wessel, Karlheinz Essl, Georg Hajdu, Schliengen, 2017, S. 76ff.

6 Vgl. o. A. "VAX 11/780, The first VAX system", <www.old-computers.com/history/detail.asp?n=20\&t=3> (18.09.2018); sowie Steve Leibson, "Using Performance Metrics to Select Micropocessor Cores for IC Designs", in: Electronic Design Automation for IC System Design, Verification, and Testing, hrsg. von Luciano Lavagno u. a., Boca Raton 2017, S. 205.

7 Vgl. Curtis Roads, The Computer Music Tutorial, Cambridge 1996, S. 789.

8 Vgl. Barry Vercoe, "New Dimensions in Computer Music. Trends and Perspectives in Signal Processing”, in: Focus 2/2 (1982), S. 15-23.

9 Vgl. ebd., sowie Peter Elsea, "Computer Music (So Far)", unter <http://artsites.ucsc.edu/EMS/Music/equipment/ computers/history/history.html> (15.01.2020) und Roads, The Computer Music Tutorial, S. 807. 
Parallel zu den Software-Entwicklungen wird in den 1980er Jahren auch an Hardwarelösungen gearbeitet, denn, so schreibt der Leiter damalige des MIT Experimental Music Studios Barry Vercoe 1982:

„A hardware audio processing system that is sufficiently general for all audio processing research while fast enough to operate in real time is still largely a dream.."10

Ein Ansatz, um dieser Herausforderung zu begegnen, war die Entwicklung von speziellen Klangprozessoren, die, gesteuert über eine andere Recheneinheit, Klänge in Echtzeit generieren können. Am IRCAM entstand beispielsweise eine ganze Serie von Klangprozessoren. Letzter und leistungsstärkster Prozessor der Serie war 1981 der sogenannte 4X. Er basierte auf einer Technologie, die ursprünglich für das Militär entwickelt wurde und konnte als einer der ersten Prozessoren parallel mehrere Berechnungen ausführen. Bei dem $4 \mathrm{X}$ handelte es sich um einen reinen Klangprozessor, der nur in Kombination mit anderen Recheneinheiten genutzt werden konnte. Am IRCAM war der 4X daher in die sogenannte IRCAM Musical Work Station eingebunden. ${ }^{11}$

Mit dem Aufkommen der weitaus günstigeren Personal Computer (PC) mit guter Rechenleistung und vor allem durch die darauf ausführbaren, ab den 1990er Jahren auch nichtinstitutionellen Nutzer*innen zugänglichen echtzeitfähigen (Musik-)Programmiersprachen, die nun zudem zumeist auch nicht mehr an eine bestimmte Hardware gebunden waren, wurde die Verwendung digitaler Technologien im Musikbereich für eine breitere Masse an Interessierten möglich. ${ }^{12}$ Dies führte zu einer Loslösung der Verwendung bestimmter Technologien von Institutionen und damit zu einem leichteren Zugang zu den entsprechenden Technologien auch für Komponistinnen, die nicht an Institutionen arbeiteten. Im Gegenzug erforderte dieser neue Zugang auch entsprechendes Wissen über die Nutzung der Technologien und dementsprechend grundlegende Kenntnisse der Software und Programmiersprachen.

Mit den aktuellen technischen Gegebenheiten ist die Rechenzeit heute, zu Beginn der 2020er Jahre, kein Hindernis mehr und erlaubt auch die sofortige Berechnung komplexer Klangsyntheseprozesse. In der nachfolgenden Betrachtung steht die Nutzung des Computers

10 Vercoe, "New Dimensions" (wie Fn. 8), S. 18.

11 Die Technologie des 4X wurde nach Fertigstellung der ersten Version durch Guiseppe di Giugno 1981 vom IRCAM an Sogitec für eine kommerzielle Weiterentwicklung übergeben. Die neue Version wurde 1984 vorgestellt. Vgl. Giuseppe Di Giugno, Jean Kott und Andrew Gerzo, „Progress Report on the 4X Machine and Its Use", in: Proceedings of International Computer Music Conference, North Texas State University 1981, <http:// hdl.handle.net/2027/spo.bbp2372.1981.029>; sowie Emmanuel Favreau, Andrew Gerzso und Patrick Potacsek, "Programmation du Processeur Numerique Temps Reel 4X", in: Proceedings of the International Computer Music Conference, Paris 1984, S. 127-129, <http://hdl.handle.net/2027/spo.bbp2372.1984.016> (beide 22.08.2020). Die Hoffnung, das Produkt so in eine kommerziell erfolgreiche Serienproduktion zu bringen, was am IRCAM selbst nicht möglich war, erfüllte sich nicht; der 4X wurde nie in größerer Stückzahl hergestellt und vermarktet.

12 Z. B. Veröffentlichung von Pure Data und Supercollider im Jahr 1996, Vertrieb von MAX über Cycling'74 ab 1997 (vgl. Akkermann, Zwischen Improvisation und Algorithmus, wie Fn. 5, S. 100ff. sowie James McCartney, "SuperCollider: A New Real Time Synthesis Language", in: Proceedings of the International Computer Music Conference ICMC, 1996, S. 257-258). 
als Klangerzeuger im Sinne eines Musikinstruments im Fokus. ${ }^{13}$ Erfüllt der "Computer" überhaupt die Definition eines Musikinstruments? Um dies diskutieren zu können, sollen vorab zwei Aspekte betrachtet werden: Was genau ist "ein Computer"? Und: Was definiert ein "Musikinstrument"?

\section{Vorüberlegungen}

\section{Was ist ein "Computer"?}

Unter dem Begriff "Computer" wird zumeist das Komplettset aus Hardware und Software verstanden, das sehr allgemein über die Funktionsweise definiert wird. ${ }^{14}$ Die Tatsache, dass es sich dabei immer um eine Kombination aus mehreren zusammenspielenden Komponenten handelt, ist zwar für die grundsätzliche Benutzung eines Computers nicht zwangsläufig wichtig, ein genauerer Blick auf dieses Set "Computer" ist jedoch durchaus lohnend. So hält es gerade aus analytischer Sicht für die Musikwissenschaft einige Herausforderungen bereit: nämlich einen zumindest in Teilen digitalen Untersuchungsgegenstand, dessen Betrachtung bekannter, aber ebenso neuer digitaler Methoden bedarf.

In den Anfängen der digitalen Klangsynthese ist die Hardware oft maßgeblich für den resultierenden Klang verantwortlich, z. B. aufgrund des verwendeten Klangprozessors oder SoundChips, auf dem gerechnet wurde. ${ }^{15}$ Die Steuerung erfolgte mittels allgemeiner Programmiersprachen oder einer speziell dafür entworfenen Software, die, je nach Entwicklungsstand, mehr oder weniger Möglichkeiten bot, um in die angelegten Prozesse einzugreifen. Mit Entwicklung der Musikprogrammiersprachen ab den 1980er Jahren und deren (mehr oder minder problemlosem) Einsatz zur direkten Berechnung von Klängen in Echtzeit ab den 1990er Jahre kann die Klangsynthese in ihrer Qualität auch von den genutzten Programmiersprachen beeinflusst werden. ${ }^{16}$ Hinzu kommt, dassbisindie2000erJahreSoftwareoftparalleloderin (wechselseitiger)Abhängigkeit mit den neu vorgestellten Hardware-Technologien entwickelt wurde - ein Wechselspiel, das sich in den auf Entwicklung von Musiktechnologien spezialisierten Bereichen durchaus erhalten hat. Heute existieren verschiedenste Programme, um Klänge zu erzeugen und zu bearbeiten. Die Klangerzeugung bzw. Einflussnahme auf die Klanggestaltung ist hierbei maßgeblich von der

13 Die Betrachtung fokussiert hierbei die Technologien bis zur Klangerzeugung bzw. speziellen Soundkarte ohne Berücksichtigung der Ausgabe-Technologien wie Lautsprecher oder Sound System.

14 „Definition of Computer: one that computes; specifically: a programmable usually electronic device that can store, retrieve, and process data" (Artikel "computer", in: The Merriam-Webster.com Dictionary, <https://www.merriamwebster.com/dictionary/computer>, 15.12.2019); siehe auch Artikel "computer", Cambridge Dictionary, Cambridge University Press 2019, <https://dictionary.cambridge.org/dictionary/english/computer> (15.12.2019).

15 Besonders hervorstechend sind hier die prägnanten Klänge der 8-Bit Sound Chips, die in Computerspielen eingesetzt wurden (vgl. Karen Collins, Game Sound. An Introduction to the History, Theory, and Practice of Video Game Music and Sound Design, Cambridge 2008, S. 20ff.).

16 Vgl. u. a. Miller Puckette, "The MAX Real-Time Computer Music System“ in: Actes du Symposium Systèmes Personnels et Informatique Musicale, Paris 1986, o. S., sowie James McCartney, "SuperCollider" (wie Fn. 12). 
verwendeten Software sowie deren Design und Steuerungsmöglichkeiten beeinflusst und reicht von eigenständigen Synthesizer-ähnlichen Programmen bis hin zu Open-Source-Musikprogrammiersprachen. Die Hardware ist dagegen - jedenfalls was das Errechnen des Klangresultats anbelangt - zumeist weniger dominant und universal (auch) für andere Aufgabengebiete einsetzbar. Grenzen werden hier eher durch die Kompatibilität von Software und Hardware gesetzt, die sich insbesondere bei den neuesten Geräteformaten wie Tablet-Computer oder Smartphones zeigen.

Diese ineinandergreifende Anlage von Hardware und Software ist nun in mehrfacher Hinsicht spannend: Sind Computer in der Einheit aus Hard- und Software zu denken, oder kann ein Bestandteil unter einer bestimmten Perspektive dominieren? Wie kann das Verhältnis der beiden Teile zueinander je nach Beispiel beschrieben werden? Müssen für eine korrekte Angabe zum "Computer" immer beide Bestandteile genannt werden? Welche Rolle spielt hierbei das User Interface, welches ebenfalls sowohl hard- als auch softwareseitig (oder auch beidseitig) angelegt sein kann? Und welche Zusammenstellung wird betrachtet, wenn vom Computer als Klangerzeuger oder gar Musikinstrument gesprochen wird?

Was definiert ein "Musikinstrument"?

Ebenso wie die Frage, was als Computer bezeichnet wird, ist auch die Frage, wie ein Musikinstrument definiert ist, nicht einfach zu klären. Zwar gibt es ausführliche Abhandlungen über die Geschichte, Entwicklung und Klassifizierungen von einzelnen Musikinstrumenten und -gruppen, eine allgemeine Definition ist jedoch, so scheint es, ebenso trivial wie wenig hilfreich, wie Tellev Kvivte es in seinem Aufsatz "What is a musical instrument" darlegt. Ausgangspunkt seiner Überlegungen ist die Definition aus Grove Music Online, wie sie im Jahr 2008 zu lesen war:

"'Musical instrument' is a self-explanatory term for an observer in his own society; it is less easy to apply on a worldwide scale because the notion of music itself in such a wide context escapes definition. [...]"17

Dies, so Kvivte, binde das Konzept des Instruments an das Konzept von Musik, und damit sei ohne eine Definition von Musik auch die Definition eines Musikinstruments nicht möglich. Während Kvivte nun mehrere Definitionen u. a. von Hornbostel, Lysloff und Matson, Bielawski, sowie Herbert Heyde zusammenträgt und resümiert, dass verschiedene Perspektiven und Interessen unterschiedliche Definitionen hervorbringen, ${ }^{18}$ umkreist Laurence Libin in seiner im

17 Tellev Kvivte, Artikel „Instruments, classification of", in: Grove Music Online, hrsg. von Laura Macy, <http://www. grovemusic.com> (23.01.2008), zitiert nach: Tellev Kvivte, "What is a musical instrument?", in: Svensk Tidskrift for Musikforskning 2008, sowie unter <https://www.researchgate.net/publication/234037743_What_is_a_musical_instrument> (07.01.2020).

$18 \mathrm{Vgl}$. ebd. 
Jahr 2014 erschienenen neuen Fassung des Grove-Online-Eintrags zu "Musical instrument" das Objekt näher und beschreibt es als

"[V]ehicle for exploring and expressing musical ideas and feelings through sound. Practically anything that is used to make sound can be employed in music [...]. Conventionally the term refers to implements specially designed for producing sound, but this definition is inadequate because unaltered natural objects as well as utensils meant for other tasks (nowadays including electronic communication devices) have been put to musical use since prehistoric times. It can be difficult to distinguish an ,instrument from a practice of music-making". ${ }^{19}$

Libin nennt hierin zwar als Kriterium auch das spezielle Design des Objekts für die Klangproduktion, verwirft diese Einschränkung jedoch gleich wieder. Damit schließt er den Computer als Musikinstrument weder eindeutig ein noch aus, insofern ein musikalischer Gebrauch vorliegt. Dies legt nahe, die Frage etwas umzuformulieren: Welche Funktionen müssen gegeben sein, damit etwas als Musikinstrument verwendet werden kann?

Ein zentrales Element bilden Steuerung und Feedback, die eine Kontrolle der Klänge und damit überhaupt erst ein gezieltes musikalisches Spielen erlauben.

Edgar Berdahl betrachtet in erster Instanz das Feedback, das gegeben sein muss, um gemeinsam musizieren zu können. Die Grundlage bilden die traditionellen Musikinstrumente, bei denen ein visuelles, auditives und haptisches Feedback die Kontrolle der mechanischen Steuerung des Instruments erlaubt (Abb. 2). Das Instrument ist hierbei eine Gesamtheit.

Fig. 9.1 When a performer plays a traditional musical instrument, he or she receives auditory, visual, and haptic feedback. The performer integrates information together from these "multisensory" feedback channels [15, 39] while giving a mechanical excitation back to the musical instrument in response

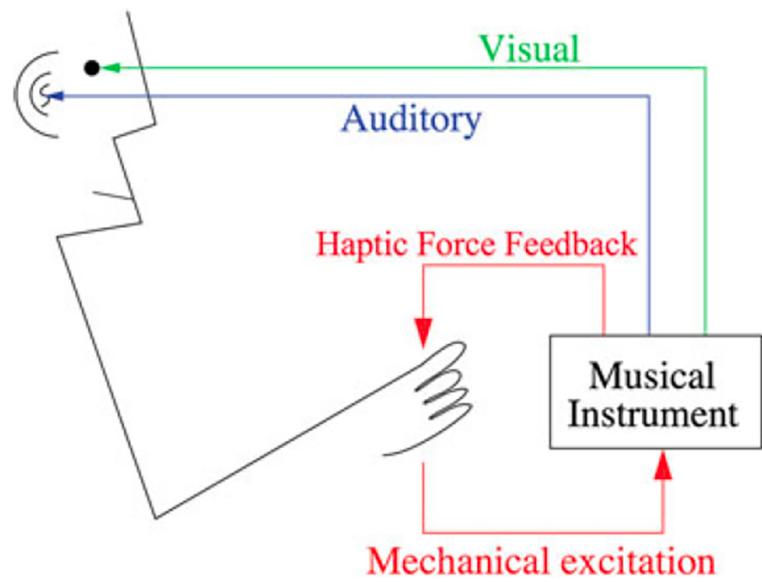

Abbildung 2: Feedback beim Spielen eines traditionellen Musikinstruments (Berdahl, „Force-Feedback Instruments”, wie Fn. 20, S. 173)

Berdahl nutzt dieses Schema für Überlegungen zu Aufführungen mit einem Laptop-Orchester, insbesondere zur Frage, wie notwendig ein haptisches Feedback für die Kontrolle der Steuerung ist. "Laptops" werden hier von Berdahl als Digital Musical Instruments bezeichnet und

19 Laurence Libin, Artikel "Musical instrument", Grove Music Online, <https://doi.org/10.1093/omo/978156159263 0.013.3000000097> (19.09.2019). 
nach Vorbild des bereits etablierten Princeton Laptop Orchestra (PLOrk) handelt es sich beim 2011 gegründeten Laptop Orchester of Louisiana heute um zehn performance stations und einen Server, wobei jede performance station selbst einen Laptop, Soundkarte, Lautsprecher mit Verstärkung und eine variierende Anzahl und Art von Controllern umfasst. ${ }^{20}$

Eduardo Miranda und Marcelo Wanderly, die sich auch mit der Frage nach Steuerung und Feedback beschäftigten, tun dies mit einem etwas allgemeineren Blick auf Digital Musical Instruments. Sie trennen in ihrer Betrachtung Steuereinheit und Klangerzeugung (siehe Abb. 3) - eine Trennung, die bei akustischen Musikinstrumenten in der Regel physisch nicht möglich ist, die jedoch gerade mit Blick auf die Einbettung von Computern sehr hilfreich sein kann. ${ }^{21}$

Figure 1.1 A possible approach to the representation of a digital musical instrument.

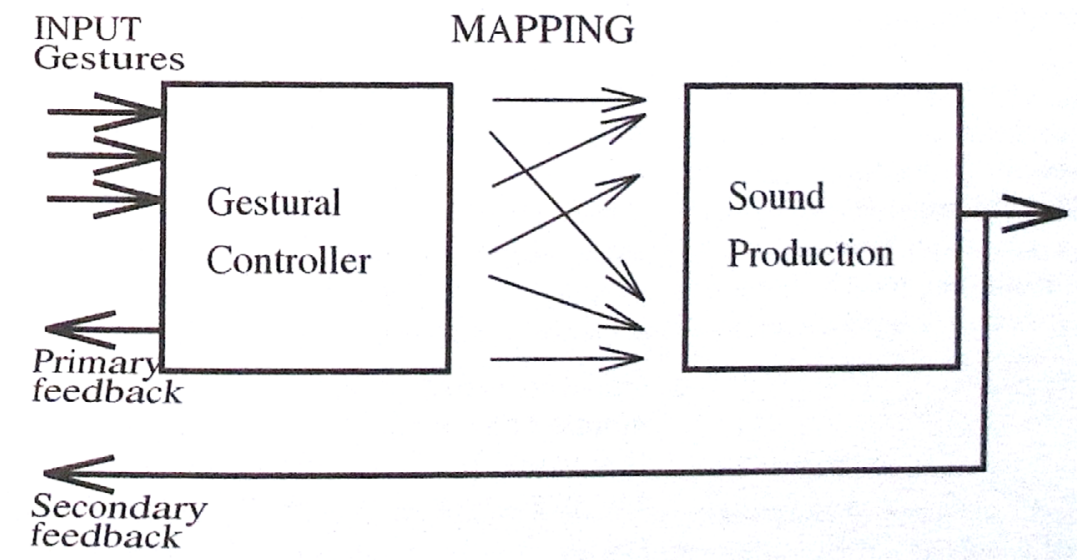

Abbildung 3: Mögliche Zusammenstellung von Kontrolleinheit und Klangeinheit in einem Musikinstrument (Miranda und Wanderly, New Digital Musical Instruments, wie Fn. 21, S. 3)

Folgt man nun dieser Systematik, so ergeben sich daraus einige Kriterien, von denen anzunehmen ist, dass sie auf Musikinstrumente zutreffen:

a) Ein Musikinstrument hat eine definierte Steuerungseinheit, mit der das (gesamte) System kontrolliert werden kann.

b) Die Art der Steuerung (und ggf. das Interface) ist für ein bestimmtes Instrument definiert (auch wenn die Steuerung oder das Interface prinzipiell verändert werden kann).

c) Es gibt eine Einheit, die den Klang generiert und deren Art der Klangproduktion definiert ist.

20 Vgl. Edgar Berdahl u. a. „Force-Feedback Instruments for the Laptop Orchestra of Louisiana“, in: Musical Haptics, hrsg. von S. Papetti und C. Saitis, Cham 2018, S. 171-191, hier S. 175.

21 Vgl. Eduardo R. Miranda und Marcelo M. Wanderley, New Digital Musical Instruments: Control and Interaction Beyond the Keyboard, Wisconsin 2006, S. 1-18. 
d) Es gibt ein Mapping, also eine Zuordnung von Steuerung/Interface zu produziertem Klang.

e) Es wird sichergestellt, dass es ein (zumeist akustisches, sonst visuelles oder haptisches) Feedback gibt, mittels der die Musiker*in ihre Aktionen überprüfen kann.

Überträgt man diese Kriterien beispielsweise auf eine Blockflöte, so wären die Steuerungseinheit bzw. das Interface die Tonlöcher, die Art der Kontrolle die Griffe, die Klangerzeugung die schwingende Luftsäule sowie die Art und Weise, wie diese in Schwingung gebracht wird, und das Feedback zum einen das Gefühl der Finger auf den Tonlöchern bzw. der Blick beim Spielen auf die Finger (primary feedback) und der erklingende Ton, der direkt hörbar ist (secondary feedback).

Wird dies nun auf ein digitales Hardware-Software-System übertragen, so gibt es verschiedenste Arten von Eingabemöglichkeiten, sowohl hardware- (z. B. Maus, MIDI-Keyboard, aber auch MIDI/OSC-Controller in verschiedenen Formen) wie auch softwareseitig (z. B. Ableton Live, OSC Control, Lemour App), um die ebenso unterschiedlichen Arten von Klangproduktion zu steuern, die von Synthese-Software über Musikprogrammiersprachen mit KlangsytheseMöglichkeiten (z. B. MAX MSP, SuperCollider, CSound) bis hin zu speziellen Klangprozessoren (z. B. 4X) reichen. Bleibt man bei den traditionellen Musikinstrumenten als Vorlage hinsichtlich ihrer Nutzung bzw. Spielbarkeit, so kommen zwei weitere Voraussetzungen hinzu: zum einen, dass die Berechnung und Kontrolle des Klanges in Echtzeit geschehen und zum anderen, dass die Zuordnung von Kontrolle zu Klang, das sogenannte Mapping (zumindest für einen bestimmten Zeitraum, ein Projekt, eine Komposition o. Ä.) unveränderlich ist. Letztgenanntes ist auch die Voraussetzung, um die Bedienung, also das Spielen eines Instruments, erlernen und üben zu können. Ist es bei traditionellen Musikinstrumenten in der Regel nicht oder nur schwer möglich, dieses Mapping zu verändern, so ist es bei vielen digitalen Kontroller-KlangerzeugerKombinationen genau andersherum: Das Mapping (und oft die gesamte Zusammenstellung der Hard- und Software) ist nur für einen spezifischen Kontext definiert. Die Art der Klänge wie auch das Mapping sind jedoch innerhalb der Zusammenstellung nicht festgelegt und werden oft auch den Bedürfnissen entsprechend verändert oder weiterentwickelt.

Die Idee, eine Komposition auf die Möglichkeiten des Instruments abzustimmen, kann hierbei neu ausgelegt werden; so setzt nicht zwangsläufig das Musikinstrument die Grenzen für das spieltechnisch Mögliche, sondern das Instrument selbst kann in die Komposition einbezogen und in Klangeigenschaft oder Spielverhalten mitkomponiert werden.

\section{Der Computer als (komponiertes) Instrument?}

Dies eröffnet neue künstlerische Möglichkeiten, so können nicht nur Spielanweisungen in Kompositionen einfließen, es können auch passgenaue Klänge auf Instrumente aufgespielt werden, die diese sonst vielleicht nicht umfassten, oder Controller vorausgesetzt werden, die individuell 
für eine bestimmte Komposition passen. Diese große Vielfalt und die weitreichenden Einflussmöglichkeiten der Komponierenden führen auf analytischer Seite zu neuen Debatten: Wann bzw. wie lange kann von einer Komposition gesprochen werden? Ab wann macht es Sinn, das Set-up als komponiertes Instrument zu betrachten? Oder handelt es sich gar um ein komponiertes Performance Environment? Was genau ist für den Klang maßgeblich und kann überhaupt noch als "das" Musikinstrument benannt werden? Wie fließend die Grenzen hierbei sein können, wird im Folgenden kurz an drei Beispielen gezeigt.

\section{David Wessel: SLABS}

Ab 2009 wurde mit und für den Musiker, Komponisten und Programmierer David Wessel am CNMAT die aus Controller und MAX-Patch bestehende Kombination SLABS entwickelt, die Wessel als digitales Musikinstrument präsentierte und auch in Konzerten und für Komposi-

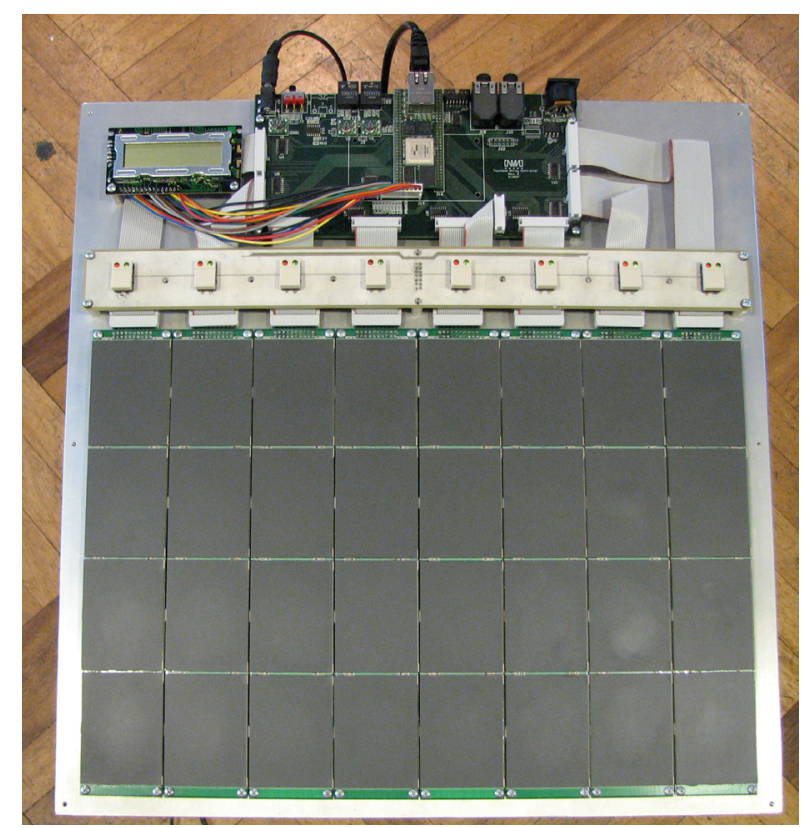

Abbildung 4: SLABS (Foto: M. Akkermann) tionen nutzte. Bereits im ersten Jahr der Entwicklung wurde Wessel dafür mit dem 3. Preis des Guthman Competition for New Musical Instruments ausgezeichnet. ${ }^{22}$ Gleichwohl ist die Zusammensetzung dieses innerhalb des Wettbewerbs als Musikinstrument anerkannten SLABS nicht eindeutig festlegbar.

Das Musikinstrument besteht aus der zweiten und letzten Variante des Controllers Slabs, der 32 druckempfindliche Touch-Pads von Sony umfasst, die mit Audio-Samplerate abgetastet werden (siehe Abb. 4). Die hieraus gewonnenen Informationen über $\mathrm{x}$ - und $y$-Position einer Berührung sowie dem entsprechenden Druck auf dem einzelnen Pad werden per OSC-Protokoll an einen Computer übergeben, auf dem dann die Klangerzeugung in einem MAX-Patch erfolgt. Wessel nutzte hierzu mehrere unterschiedliche Patches, denen je nach Nutzungszweck oder intendierter Komposition ein spezielles Mapping und entsprechende Klänge zugrunde lagen..3 Das Mapping ebenso wie die Klangsynthese wird vollständig auf einem Laptop berechnet, der Controller selbst beinhaltet keine eigene

22 Vgl. „News", in: Computer Music Journal 33/3 (2009), S. 6. Bei dem Wettbewerb, der vom Georgia Tech Center for Music Technology ausgeschrieben wird, werden jedes Jahr neu entwickelte digitale Musikinstrumente ausgezeichnet.

23 Vgl. David Wessel, „SLABS: Arrays of Pressure Sensitive Touch Pads”, <http://cnmat.berkeley.edu/user/david_ wessel/blog/2009/01/15/slabs_arrays_pressure_sensitive_touch_pads> (11.11.2013), sowie Akkermann, Zwischen Improvisation und Algorithmus (wie Fn. 5), S. $102 \mathrm{ff}$ und $127 \mathrm{ff}$. 
Recheneinheit. Die Besonderheit dieser Instrument-Kombination ist die schnelle Datenübertragung zwischen Controller und Recheneinheit, die ein latenzfreies und somit direktes Steuern der erzeugten Klänge über drei Parameter pro Touch-Pad auf mehreren Pads gleichzeitig erlaubt. Zudem ermöglicht die freie Zuweisung der Parameter eine individuelle Konfiguration des Controllers. ${ }^{24}$ Diese große Freiheit zieht aus analytischer Sicht nun die Frage nach sich, welche Elemente eigentlich zu dem Musikinstrument SLABS gehören: Neben dem HardwareInterface und dem Computer spielen die verwendeten MAX-Patches eine entscheidende Rolle, wird mit ihnen doch Kontrollverfahren/Spielmechanik, Mapping und Klang festgelegt. Für ein Musikinstrument sollten diese Elemente verlässlich festgelegt sein, um eine gezielte Nutzung zu ermöglichen. Für den SLABS gilt dies nicht. Wessel konfiguriert das Instrument für jedes seiner Stücke, die er darauf spielt, neu. Zwar hebt er selbst hervor, dass für ihn ein Musikinstrument nur dann sinnvoll gestaltet ist, wenn dieses geübt und das Spiel darauf perfektioniert werden kann, dies trifft auf SLABS jedoch nur innerhalb einer Komposition zu. Dies provoziert die Frage, inwieweit die Patches zum Instrument oder zur Komposition gehören. Wie soll mit den Patches, also der speziellen Software verfahren werden? Und wie kann eine Zusammenstellung wie SLABS überhaupt eingeordnet oder analysiert werden? Bei der Erarbeitung solcher Kombinationen forschen oft Programmierer mit Musikinteresse neben Komponisten mit guten Programmierkenntnissen. Eine klare Trennung der Bereiche ist kaum mehr möglich - und meist auch nicht mehr sinnvoll. Auch in der Untersuchung ist daher eine enge Zusammenarbeit verschiedener Wissensbereiche nötig.

Wie eng diese Bereiche beieinander liegen, wird gerade an letztgenannter Frage deutlich, also in der Debatte darüber, wer über den Klang und die Bedienung des Instruments entscheidet. Wessel hat, in seiner Personalunion von Entwickler, Komponist und Musiker, Einfluss auf alle Bereiche. SLABS ist von ihm jedoch, auch wenn es in der Betrachtung sehr zu verschwimmen scheint, immer als Musikinstrument gedacht und auch konzipiert worden. In der systematischen Untersuchung liegt die Herausforderung, zwischen Instrument und Komposition zu differenzieren: z. B. im Detail des jeweiligen Einsatzes durch Wessel, wobei Teile des Instruments, der Controller SLABS, das Instrument eindeutig kennzeichnen. Noch stärker verschwimmen die Grenzen, wenn die verwendeten Controller nicht mehr signifikant sind und die Steuerung (theoretisch) auch komplett softwareseitig vonstatten gehen kann.

Karlheinz Essl: m@ze²

Karlheinz Essl entwickelte für sich ein instrumentartiges Set-up, das primär durch die Software gekennzeichnet wird. Das von 1998-2016 entstandene "m@ze²" ist in Max geschrieben und besteht aus einer Kombination verschiedener Bausteine, die Essl selbst als "computer-based electronic instrument which serves as a realtime environment for composition and improvisa-

24 Wessel, "SLABS" (wie Fn. 23). 
tion"25 bezeichnete. Das MAX-Patch (Abb. 5) kann mit verschiedenen externen Controllern, aber auch als reines Software-Instrument benutzt werden. Essl implementiert darin auch seine Real Time Composition (RTC) Library, die eine Sammlung an MAX-Patches und Erweiterungen enthält und die ursprünglich für Essls Komposition Lexikon-Sonate erarbeitet wurde. ${ }^{26}$

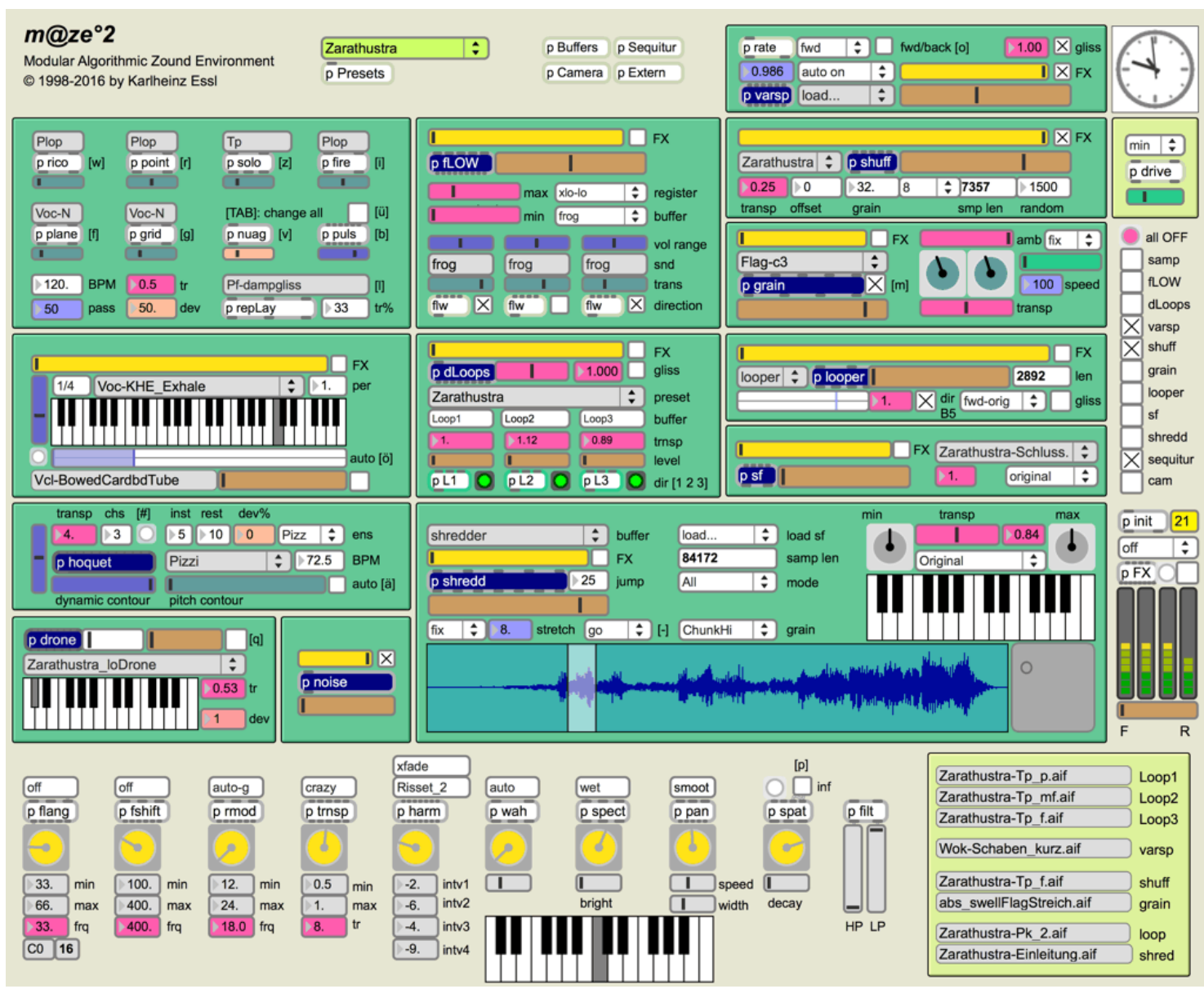

Abbildung 5: Screenshot Userinterface von m@ze 2

(Karlheinz Essl, <http://www.essl.at/works/maze.html>, 19.09.2019)

Entgegen der Konzeption des SLABS, bei dem der Controller SLABS für das Instrument kennzeichnend ist, ist die Hardware für die Zusammensetzung von Essls Instrument zweitrangig; die Art und die Anzahl der angeschlossenen Controller variiert je nach Einsatz und Essels Wünschen. Neben unterschiedlichen MIDI-Controllern kommt beispielsweise auch Bewegungssteuerung zum Einsatz. ${ }^{27}$

25 Karlheinz Essl, „m@ze ${ }^{\circ}$ ", <http://www.essl.at/works/maze.html> (19.09.2019).

26 Vgl. Karlheinz Essl, „RTC-lib”, <http://www.essl.at/works/rtc.html> (07.01.2020), sowie Miriam Akkermann, "Instrument+", in: Neue Zeitschrift für Musik 178/2 (2017), S. 31-33.

27 Siehe hier z. B. eine Performance, die im Rahmen eines Portraitkonzerts von Essl im Projects Art Centre in Dublin im Oktober 2011 entstand (Vgl. Karlheinz Essl, m@ze²: solo performance Karlheinz Essl, <www.youtube.com/ watch?time_continue $=87 \& v=A 7 t G B h r l s D g>$, 19.09.2019), sowie die Beschreibung von Essl zu "m@ze²" (wie Fn. 25), 
Wie die Steuerung, so ist auch die Klangsynthese veränderbar. Essl verwendet wie Wessel passende Voreinstellungen für unterschiedliche Anwendungen, so gibt es Presets für Kompositionen, z. B. ein an die Spielangaben angepasstes Preset für Essls Komposition more or less, bei der bereits die in der Komposition beschriebenen Klangtexturen unter entsprechenden Begriffen hinterlegt sind. ${ }^{28}$

Blickt man auf das so entstehende Instrument, so zeigt sich, dass hier die Software das zentrale Element bildet, während sowohl Controller als auch Recheneinheit angepasst werden können. Das Instrument wird damit mehr durch die softwareseitige Steuerung und die damit verbundene Klanggenese als durch eine physische Steuerung gekennzeichnet. Dies hat auch Auswirkungen auf die ideelle Trennung von Instrument und Komposition: Wessel denkt Komposition und Instrument als verschiedene Einheiten, auch wenn er selbst den SLABS in den Klangeigenschaften variiert und für Improvisationen individuell konfiguriert. Der SLABS wird als Instrument verstanden, der auch von anderen Performer*innen verwendet und/oder programmiert werden kann. Bei Essl stehen Instrument und Komposition sehr viel näher und in einem Environment zusammen; "m@ze ${ }^{\circ}$ " wurde von und für Essl selbst entworfen und ist auch nicht für eine freie Verwendung angelegt.

Je mehr die Software ins Zentrum rückt, umso mehr kommt auch das Komplettset Computer in den Fokus, denn mit fehlenden Controllern wird die Hardware des Computers zum physischen Erscheinungsbild des neuen Musikinstruments, das nun verschiedenste Instrumente darstellen und ausführen kann.

Shelly Knotts: Live Coding Supercollider

Besonders deutlich wird dies, wenn die Klang-Programmierung in Echtzeit und ohne weiteres Interface vonstatten geht, wie beispielsweise beim sogenannten Live Coding. Hier ist der Computer, zumeist ein Laptop, und die für die Programmierung verwendete Programmiersprache je nach Blickwinkel Instrument, Controller und Klanggenerator in einem - oder nichts davon.

Shelly Knotts, eine britische Komponistin, Performerin und vor allem Live Coderin schreibt Kompositionen für vernetzte Musiker*innen und Live Coding. Sie verwendet dafür zumeist Supercollider, eine textbasierte Musikprogrammiersprache, und führt diese Kompositionen z. B. mit Algosix, einer Gruppe von sechs Musikerinnen, die Solo-Auftritte online live streamen, selbst auch live auf. Die Musikerinnen sitzen hierbei an Spielorten über die ganze Welt verteilt und performen/programmieren Klang in Echtzeit. Die Performances dauern bis zu 72 Stunden und setzen sich, ähnlich wie in einem Club, aus aufeinanderfolgenden Sets zusammen, die vom jeweiligen Spielort per Internet an die anderen Spielorte gestreamt werden. Die Performances

28 Vgl. Karlheinz Essl, "m@ze2” (wie Fn. 25). 
sind somit zeitgleich live an mehreren Orten zu hören. ${ }^{29}$ Die Arbeit schafft eine Brücke zwischen einem experimentellen Bereich der Computermusik, dem Live Coding, und experimenteller, tanzbarer Elektronischer Musik und zeigt, wie nah sich die verwendeten Techniken in der Produktion stehen können. Die Herausforderungen des ,Instruments Computer' treten hierbei in mehrfacher Hinsicht in Erscheinung: Der von Knotts verwendete Laptop ist zwar ihr Musikinstrument, im Sinne der grundlegenden Definition eines Musikinstruments jedoch nur schwer einzuordnen. Die Vorgabe des Mappings ist über die Programmiersprache erfüllt, der Zugriff bzw. die Steuerung der Klänge erfolgt unmittelbar über die Tastatur auf Code-Ebene, eine zusätzliche graphische oder externe Steuerungs-/Bedienoberfläche fehlt jedoch, auch wenn das verwendete Programm selbst einige Steuerungshilfen bereitstellt, die ebenso musikspezifisch sind, wie die in den beiden vorangehenden Beispielen beschriebenen. Durch das Streamen der Performances kommt ein Netzwerk aus Computern ins Spiel, das zu einer indirekten Erweiterung des Instruments wird. Zwar ist es am direkten Prozess des Musikmachens nicht beteiligt, und damit streng genommen auch nicht Teil des Musikinstruments, es bietet jedoch die Aufführungsumgebung, also alle notwendige Ausstattung, um das Instrument selbst überhaupt hörbar zu machen. Dies, wie auch die Nähe zur Elektronischen Tanzmusik eröffnet nun neben der definitorischen auch eine Reihe an nachfolgenden Fragen, beispielsweise ob ein ,Computer' auch dann als Musikinstrument einzustufen ist, wenn er zur Montage von Tracks durch Musikproducer zum Einsatz kommt.

\section{(Musik)Instrument (im) Computer - Musikinstrument Computer!}

Wenn der Computer also selbst zum Instrument wird, so stellen sich nicht nur dahingehend neue Herausforderungen, wie mit diesen Gegenständen umzugehen ist, es eröffnen sich auch neue Möglichkeiten, über Musik und ihre Aufführung nachzudenken.

Die Kombination aus digitalen und analogen Elementen erfordert es, scheinbar triviale Dinge wie ein Musikinstrument (präzise) zu definieren und vorausgesetzte Inhalte oder Annahmen explizit zu machen. Die daraus erwachsenden Möglichkeiten erlauben auch eine andersartige Diskussion zwischen Wissenschaftlern und Künstlern, die sich z. B. auf technischer Ebene oder auch hinsichtlich der Archivierung digitaler Musikstücke plötzlich mit den gleichen Fragen konfrontiert sehen.

Wie steht es mit den sogenannten Software-Instrumenten, die, teils Hardware-unabhängig, den Nutzer*innen musikalisches Spiel erlauben, aber bereits viele klangliche Entscheidungen abnehmen? Wie können und sollen Interfaces eingeordnet werden, die, als Hardware-Extension speziell für die musikalische Nutzung entwickelt, doch immer noch eine (spezielle) Software

29 Siehe z. B. Shelly Knotts - Algosix Live Stream Performance - March 16, 2018 05:30 UTC in Melbourne <www. youtube.com/watch?v=Gm-EXf9hbxI $>$, sowie <https://algorave.com/wearesix $>$ und <http://datamusician.net/> (alle 19.09.2019). 
und weiteres technisches Equipment zur Klangerzeugung und vor allem -ausgabe brauchen? Was geschieht, wenn die Ausgabetechnik, z. B. Lautsprecher, auch noch mitbedacht werden? Und wer entscheidet überhaupt über den Klang und die Bedienung des neuen Instruments erfolgt die Einbindung von Computern in musikalische Arbeiten doch zumeist in enger Zusammenarbeit von Komponist*innen, Musiker*innen, Ingenieur*innen und Programmierer*innen? Um diese Fragen diskutieren zu können, ist neben neuen methodischen Ansätzen auch die Zusammenarbeit zwischen den Disziplinen eine unumgängliche Bereicherung.

Zitation: Miriam Akkermann, "(Musik)instrument (im) Computer", in: Brückenschläge zwischen Musikwissenschaft und Informatik. Theoretische und praktische Aspekte der Kooperation, in Verbindung mit der Fachgruppe Digitale Musikwissenschaft hrsg. von Stefanie Acquavella-Rauch, Andreas Münzmay und Joachim Veit (= Musikwissenschaft: Aktuelle Perspektiven. Bericht über die Jahrestagung der Gesellschaft für Musikforschung 2019 in Paderborn und Detmold, Bd. 3), Detmold, Musikwissenschaftliches Seminar der Universität Paderborn und der Hochschule für Musik Detmold, 2020, S. 125-140, DOI: 10.25366/2020.102 


\section{Abstract}

The use of the computer as a sound generator is omnipresent in current music production and ranges from music notation programs playing back samples via MIDI control to specially programmed sound synthesis programs. The term ,computer' is generally understood as a complete set of hardware and software. But a closer look at this complete set is definitely worthwhile and poses some systematical challenges. In the early days of digital sound synthesis in real time, the hardware is strongly connected to the resulting sound. The control was done by means of a programming language or a specially designed software, which offered more or less possibilities of intervention, depending on the stage of development. But do these sound generators actually fulfill the definition of a musical instrument - and what exactly is that definition? What about the so-called software instruments, which, partly hardware-independent, allow users to play music? How can and should interfaces be classified seeing that hardware extensions developed specifically for musical use, but still need (special) software and other technical equipment for sound generation and, above all, output? And who actually decides on the sound and handling of the new instrument, since the integration of computers into musical works usually takes place in close cooperation between composers, musicians, engineers and programmers? In order to be able to discuss these questions, not only new methodological approaches but also cooperation between the disciplines is unavoidable and the same time rewarding.

\section{Kurzvita}

Miriam Akkermann studierte Querflöte und "Music and New Technologies" am Conservatorio C. Monteverdi Bolzano, Komposition und Klangkunst an der Universität der Künste, sowie Produktdesign an der Freien Universität Bozen. Während ihres Studiums und der Promotionszeit erhielt sie mehrere Stipendien: vom Leo Baeck-Institute New York (2009), vom DAAD (2011), von der Elsa Neumann-Stiftung (2011-2013). Im Jahr 2014 wurde sie an der Universität der Künste Berlin mit einer Dissertation zum Thema Zwischen Improvisation und Algorithmus. David Wessel, Karlheinz Essl und Georg Haju promoviert. Ihre Kompositionen, Klanginstallationen und Performances sind auf internationalen Festivals und in Galerien vertreten. Seit 2019 ist sie Juniorprofessorin für Empirische Musikwissenschaft an der TU Dresden. 



\section{Brückenschläge zwischen Musikwissenschaft und Informatik}

Theoretische und praktische Aspekte der Kooperation

Herausgegeben von Stefanie Acquavella-Rauch,

Andreas Münzmay und Joachim Veit 
Brückenschläge zwischen Musikwissenschaft und Informatik 


\section{Musikwissenschaft: Aktuelle Perspektiven}

Bericht über die Jahrestagung der Gesellschaft für Musikforschung 2019 in Paderborn und Detmold

Herausgegeben von Rebecca Grotjahn und Nina Jaeschke

Band 3 


\section{Brückenschläge zwischen Musikwissenschaft und Informatik}

Theoretische und praktische Aspekte der Kooperation

Beiträge der Symposien zur Digitalen Musikwissenschaft

Osnabrück 2018 und Paderborn 2019

im Rahmen der Jahrestagungen der Gesellschaft für Musikforschung

In Verbindung mit der Fachgruppe Digitale Musikwissenschaft herausgegeben von

Stefanie Acquavella-Rauch, Andreas Münzmay und Joachim Veit

Detmold: Musikwissenschaftliches Seminar der Universität Paderborn und der Hochschule für Musik Detmold 2020 
DOI: $10.25366 / 2020.87$

Online-Version verfügbar unter der Lizenz: Urheberrecht 1.0, $<$ https://rightsstatements.org/page/InC/1.0/?language =de >

Bibliografische Information der Deutschen Nationalbibliothek

Die Deutsche Nationalbibliothek verzeichnet diese Publikation in der Deutschen Nationalbibliografie; detaillierte bibliografische Daten sind im Internet über http://dnb.d-nb.de abrufbar.

\section{Impressum}

Redaktion: Stefanie Acquavella-Rauch, Andreas Münzmay und Joachim Veit Satz: Nina Jaeschke und Joachim Veit

(C) Musikwissenschaftliches Seminar der Universität Paderborn und der Hochschule für Musik Detmold 2020 


\section{INHALT}

Rebecca Grotjahn, Nina Jaeschke

Vorwort zu Band 1-3

IX

Stefanie Acquavella-Rauch, Andreas Münzmay, Joachim Veit

Brückenschläge zwischen Musikwissenschaft und Informatik - Vorbemerkung

$\mathrm{XI}$

\section{KOLLABORATIONEN - KO-LABORATORIEN}

\section{Reinhard Keil}

Der Computer als Denkzeug für hermeneutische Arbeit

\section{Ulrich Konrad}

Philologie und Digitalität. Perspektiven für die Musikwissenschaft im Kontext fächerübergreifender Institutionen

\section{Gudrun Oevel}

Infrastruktureinrichtungen in Forschungsprojekten - Spagat oder Chance?

\section{Dennis Ried}

Erhebung, Transformation und Präsentation digitaler Forschungsdaten

Anna Neovesky, Frederic von Vlahovits

IncipitSearch - Leitfaden zur Zusammenarbeit

Elisabeth Treydte

Clara Schumann \#digital. 40 Jahre Archiv Frau und Musik und der Start in die Digitalisierung

\section{TEXT/DATEN/PROZESSE}

\section{Christine Siegert}

Komponisten-Gesamtausgaben im digitalen Zeitalter: Perspektiven

und Reflexionen am Beispiel Ludwig van Beethovens

Markus Neuwirth, Johannes Hentschel, Martin Rohrmeier

Perspectives of Musical Corpus Studies: The Annotated Mozart Sonatas

Agnes Amminger, Franz Kelnreiter

Leopold Mozarts „Gründliche Violinschule". Zur Textcodierung und -präsentation einer digitalen Edition

\section{Oleksii Sapov}

Algorithmische Automatisierung komplexer Notationsregeln in MEI-XML am Beispiel von Versetzungszeichen 


\section{Susanne Cox, Richard Sänger}

Digitale Fassungsvergleiche am Beispiel von Beethovens Eigenbearbeitungen

Agnes Seipelt

Digitale Edition und Harmonische Analyse mit MEI von Anton Bruckners

Studienbuch

Stefanie Acquavella-Rauch

Musikalische Schaffensprozesse 2.0 - Inkorporation audiovisueller Medien

der populären Musik in Methoden der digitalen Edition

\section{DIGITAL(ISIERT)E MATERIALITÄTEN}

\section{Miriam Akkermann}

(Musik)Instrument (im) Computer

\section{Daniel Fütterer}

Herausforderungen bei der Kodierung von Paratext am Beispiel Neuer Musik mit Live-Elektronik

\section{Matthias Pasdzierny}

How much is the glitch? Das digitale Paradigma als Herausforderung

und Chance für die historische Musikwissenschaft

\section{Shintaro Miyazaki}

Musik für Maschinen?! - Wo sich die Wissenschaft der Medien, des Computers und der Musik treffen und wie sie zusammenarbeiten könnten

\section{MUSIKGESCHICHTE(N) IM NETZ}

\section{Matthias Tischer}

Musikgeschichte der DDR: Ein Pilotprojekt zur digitalen Musikvermittlung

Annette van Dyck-Hemming, Jan Eberhardt, Melanie Wald-Fuhrmann

Ansätze zur Analyse historischer Netzwerke mit Neo4j® - Aus der Projekt-Werkstatt der Datenbank zur Fachgeschichte der Musikwissenschaft

Axel Beer, Martin Bierwisch, Kristina Krämer

Das MMM2 - Ein regionalgeschichtliches Onlinelexikon der Arbeitsgemeinschaft für mittelrheinische Musikgeschichte

\section{Matej Santi}

Was erzählt Fritz Kreislers Geige?

\section{Elias Berner}

Alle Menschen werden Brüder?! Ein historisches Dokument aus dem

Nationalsozialismus in den sozialen Medien 
Gabriele Buschmeier in memoriam 